\title{
A Multi-Level Optimization Algorithm and a Ship Design Application
}

\author{
Hong Yoon $\mathrm{Kim}^{1}$ and Nickolas Vlahopoulos ${ }^{2}$ \\ University of Michigan, Ann Arbor, Michigan, 48109, United States
}

\begin{abstract}
In this paper, a multi-level optimization algorithm is presented. The algorithm constructs a system level objective function that drives all disciplines toward each discipline's optimal point while satisfying all necessary discipline constraints and the system level constraints. Mathematical convergence of the system level optimization is demonstrated. Communication among the system and the discipline level optimizations is facilitated by the new algorithm. An analytical example, which has a non-convex Pareto front with coupled disciplines, is solved for highlighting the main capabilities of the new algorithm, and a ship design optimization analysis is conducted. The latter demonstrates how the new algorithm can be used for analyzing a complex engineering system.
\end{abstract}

\section{Nomenclature}

Design parameter

$\rho$ : a vector of discipline objective function input values, which are functions of $x$.

$\rho\left(x^{p}\right)$ : design parameter on $\mathrm{p}^{\text {th }}$ iteration of the system level optimization.

\section{Design Variable}

$x$ : a vector of input variables that the optimizer is changing to minimize the objective function.

$x_{j}$ : the $\mathrm{j}^{\text {th }}$ variable of $x$.

$x(i)$ : the $i^{\text {th }}$ discipline design variable.

$x^{p}$ : design variable on $\mathrm{p}^{\text {th }}$ iteration of the system level optimization

Optimal Point

$x^{*}(i)$ : a point where $\mathrm{i}^{\text {th }}$ discipline achieves its optimal value

$x^{* p}(i)$ : optimal point at $\mathrm{i}^{\text {th }}$ discipline on $\mathrm{p}^{\text {th }}$ iteration of the system level optimization

Objective Function

$f_{i}(x)$ : the function at $\mathrm{i}^{\text {th }}$ discipline mapping $\mathbb{R}^{n} \rightarrow \mathbb{R}$ that the optimizer is minimizing (or maximizing).

$f_{i}\left(x^{*}(i)\right):$ the $\mathrm{i}^{\text {th }}$ discipline optimal objective function value

$f_{\text {sys }}(x)$ : system level objective function.

Inequality/Equality Constraints

$g_{i}(x)$ and $h_{i}(x):$ inequality/equality for the $\mathrm{i}^{\text {th }}$ discipline optimization.

$g_{\text {sys }}(x)$ and $h_{\text {sys }}(x)$ : inequality/equality for the system level optimization.

Plausible Reduction Range

$\operatorname{prr}_{i}=\max \left(f_{i}\left(x^{*}(j)\right)-f_{i}\left(x^{*}(i)\right)\right) \forall j \backslash i$ where $x^{*}(j)$ is the optimal point for $\mathrm{j}^{\text {th }}$ discipline.

${ }^{1}$ Student, Department of Naval Architecture and Marine Engineering, 2600 Draper Dr., Ann Arbor MI 48109.

${ }^{2}$ Professor, Department of Naval Architecture and Marine Engineering, 2600 Draper Dr., Ann Arbor MI 48109.

American Institute of Aeronautics and Astronautics 


\section{Upper/Lower Bound}

$u b \& l b$ : a vector of upper/lower bound for design variable $x$.

$\left[u b_{i}^{p}=\min \left(x^{p}-\frac{\alpha}{p} R_{x}^{p}, u b_{s y s}\right) \& l b_{i}^{p}=\max \left(x^{p}+\frac{\alpha}{p} R_{x}^{p}, l b_{s y s}\right)\right]:$ upper/lower bound for $\mathrm{i}^{\text {th }}$ discipline on

$\mathrm{p}^{\text {th }}$ iteration of the system level optimization. ( $\propto=$ a constant)

$R_{x}^{p}=\max \left(x^{* p}(i)-x^{* p}(j): i \neq j, \forall i, \forall j\right)$ : the maximum difference of each design variable across the optimal discipline value.

$u b_{s y s} \& l b_{s y s}$ : a collection of largest initial lower bound (smallest upper bound) of each design variable.

Weight

$w_{i}$ : positive weights for $\mathrm{i}^{\text {th }}$ discipline for the system level objective function.

\section{Introduction}

General information on engineering design optimization is presented in Refs. 1-5. In fields, in which complex engineering systems are designed - such as naval architecture, ${ }^{6-9}$ automotive engineering, ${ }^{10,11}$ mechanical engineering, ${ }^{12-14}$ and in biomedical engineering, ${ }^{15}$ it is of particular interest to automatically synthesize the conflicting objectives from several disciplines into the search for one overall, globally optimum design. ${ }^{16-19}$ From the design optimization methods considered and proposed in the literature, Multi-discipline Design Optimization (MDO) was widely recognized at an early stage by many on the cutting edge of engineering design as the key to the future. ${ }^{20,21}$ This recognition stems from the requirement to synthesize several complex and computationally intensive disciplines into a single resultant design that comprises the optimum from the perspective of an equally complex top-level objective function. ${ }^{12,22-26}$ For guidance in using MDO, papers outline the steps in creating an MDO framework, ${ }^{27}$ discussing the characteristics of existing frameworks, ${ }^{28,29,34}$ and applying MDO in various disciplines. ${ }^{18}$ Multi-level optimization algorithms, like the ones presented in Refs. 30-32 provide a systematic way of organizing the solution and the flow of information between the separate multiple discipline and system level optimizations.

Multi-Level System (MLS) design comprises an approach in guiding the decision making process for designing a complex system where mutually competing objectives and disciplines need to be considered and evaluated. Mathematical relationships between the design variables and the multiple discipline performance objectives are developed adaptively as the various design considerations are evaluated and as the design evolves. ${ }^{33}$ These relationships are employed for rewarding performance improvement during the decision making process by allocating more resources and influence to the disciplines that exhibit the greatest improvement. The interdependency, the implied relationships, the implied variables, and the interactions that are present in a complex system are captured during the decision making process.

The new MLS algorithm that is presented in this paper constructs a system level objective function that drives all disciplines toward each discipline's optimal point while satisfying all necessary discipline constraints and the system level constraints. A Euclidean distance to a Utopia point is utilized for defining the system level objective function. Using a Euclidean distance to the Utopia point as a goodness of fit has been used by MDO researchers. ${ }^{35,36}$ Given a proper scaling, this approach can find a non-convex Pareto front, which a typical weighted sum approach cannot find. ${ }^{13}$ The algorithm presented in this paper facilitates the communication among system and discipline level optimizers, and it updates the system level and discipline level optimization statements as the system level optimization progresses. An iterative computation of objective functions not only ensures that the converged point does not violate any constraints, but it also enables the algorithm to handle any interdependencies that are present in the disciplines.

The structure and the work flow of the MLS algorithm are presented first. Mathematical convergence of the system level optimization is demonstrated, and an analytical mathematical example is solved to highlight the new algorithm's main capabilities that are described above. Finally, a general ship design optimization analysis is conducted where multiple disciplines are considered. 


\section{Operation and Mathematical Formulation of the MLS Algorithm}

Some of their key capabilities of the MLS algorithm are: the ability to converge on a non-convex Pareto front, compliance of the system-level solution with all discipline-level constraints, and the ability to capture the interdependency among disciplines. The flow of operations in the MLS algorithm is presented in Fig. 1. The optimizers iterate the process until the convergence is achieved at the system level.

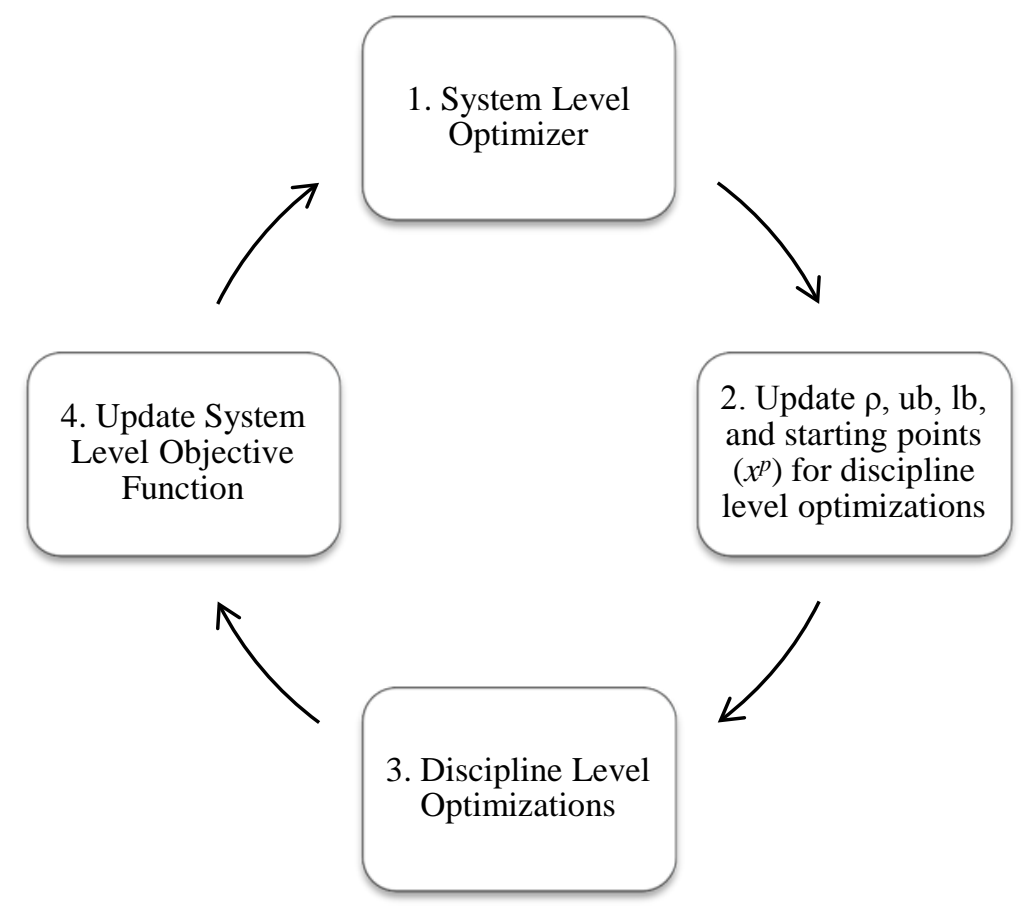

Figure 1. Flow of operations in the MLS algorithm.

The following steps are taken during the optimization process.

1. The system level optimization minimizes the scaled Euclidean distance to the Utopia point.

2. At each iteration of the system level optimization, the design parameters $(\rho)$, the upper bound $(u b)$, the lower bound $(l b)$, and the starting point $\left(x^{p}\right)$ of the discipline level optimizations are updated.

3. The discipline level optimizations are conducted, and the discipline level results are being used to update the system level objective function.

4. The system level optimization continues until convergence is achieved.

The algorithm is a multi-level structure with one system-level optimizer and many discipline-level optimizers. The flow chart of the MLS algorithm is presented in Fig. 2. The detailed description of algorithm is presented in subsequent sections. 


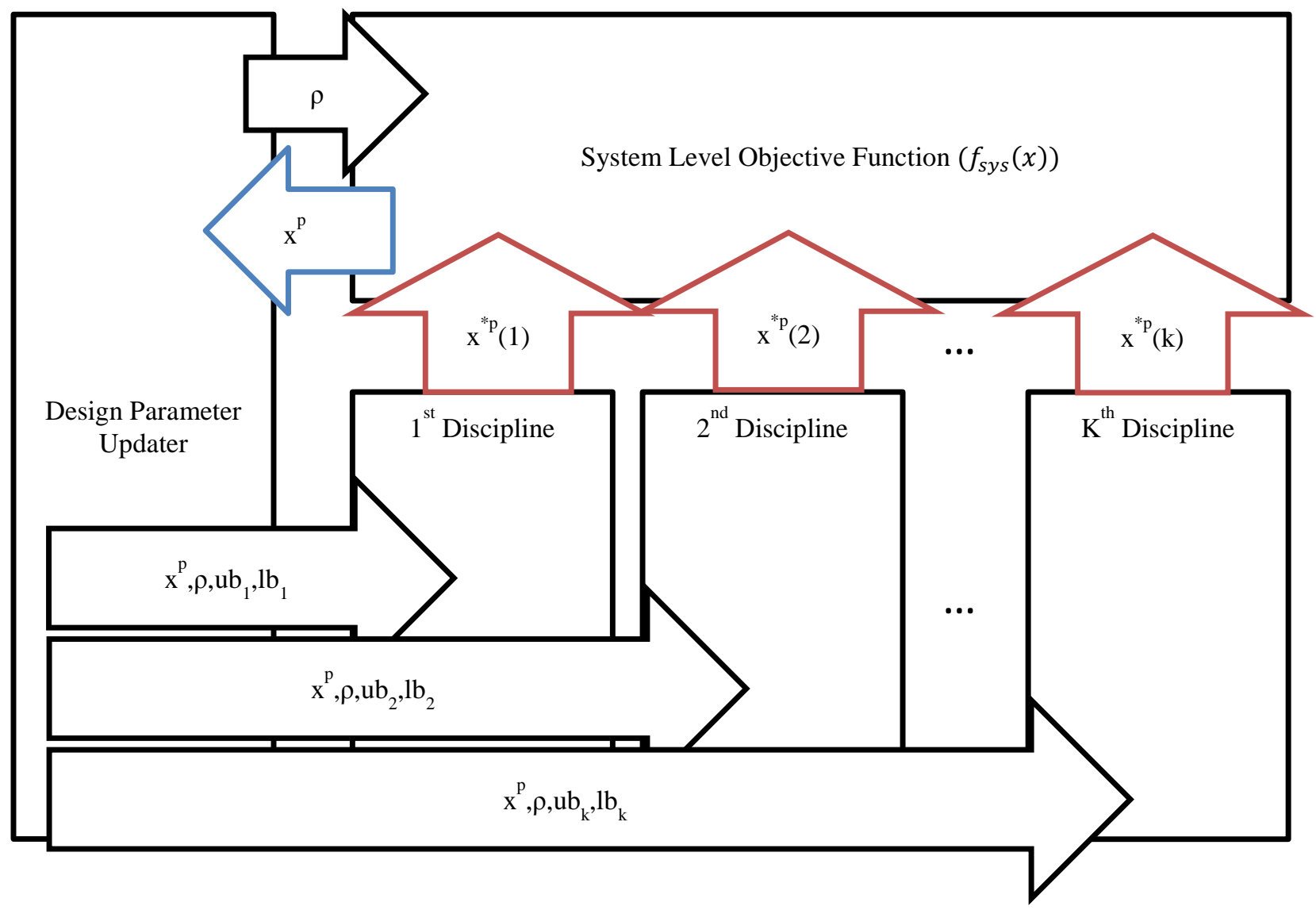

Figure 2. Flow chart of the MLS algorithm.

During each iteration of the system level optimization, the system level optimizer launches the discipline level optimizers and provides each one of them updated values for the upper bound $(u b)$, lower bound $(l b)$, design parameters $(\rho)$, and the starting point of each discipline level optimization $\left(x^{p}\right)$. Then, each discipline optimizer identifies its new optimum with updated optimization statements. Finally, the algorithm feeds the information from the discipline level to the system level to update the system level optimization. In general, the changing values of the design parameters cause each discipline level optimization to converge on different solution every time the discipline analysis is conducted. The discipline level and the system level optimization statements are presented next.

\section{A. Discipline level optimization statement}

Each discipline has its own objective function $\left(f_{i}(x)\right)$, inequality constraint $\left(g_{i}(x)\right)$, equality constraint $\left(h_{i}(x)\right)$, and upper/lower bound $\left(u b_{i}, l b_{i}\right)$. It is assumed that each discipline level optimization statement satisfies conditions A1-A6, such that each discipline statement is considered to be well-formulated for a gradient-based optimizer. ${ }^{37-41}$

$$
\begin{gathered}
\min \left(f_{i}(x)\right) \\
\text { subject to } g_{i}(x) \leq 0, h_{i}(x)=0 \\
l b_{i} \leq x \leq u b_{i}
\end{gathered}
$$

A1. $f_{i}(x)$ is bounded below

A2. $f_{i}(x)$ is second-derivative continuous (i.e., the gradient and Hessian are continuous)

A3. $g_{i}(x)$ is second-derivative continuous (i.e., the gradient and Hessian are continuous)

A4. $h_{i}(x)$ is second-derivative continuous (i.e., the gradient and Hessian are continuous)

A5. $\left|g_{i}(x)\right|<\infty \&\left|h_{i}(x)\right|<\infty$ for $\left\{x: l b_{i} \leq x \leq u b_{i}\right\}$

A6. There exists a feasible solution 


\section{B. System level optimization statement}

The system level objective function $\left(f_{\text {sys }}\right)$ is a scaled Euclidean distance to the Utopia point. Each discipline is scaled by its Plausible Reduction Range (prr) and the weights assigned to each discipline $\left(w_{i}\right)$. prr is the maximum difference of each discipline's optimal objective function value $\left(f_{i}\left(x^{*}(i)\right)\right)$ and the function evaluated at all other discipline's optimal points $\left(f_{i}\left(x^{*}(j)\right)\right)$.

$$
\operatorname{prr}_{i}=\max \left(f_{i}\left(x^{*}(j)\right)-f_{i}\left(x^{*}(i)\right)\right) \forall j \backslash i \text { where } x^{*}(j) \text { is the optimal point for } \mathrm{j}^{\text {th }} \text { discipline. }
$$

This type of scaling is shown to find a non-convex Pareto front. ${ }^{13}$ By assigning various weights in the system level objective function, the algorithm can control the convergence on the Pareto front.

The inequality constraint $\left(g_{s y s}(x)\right)$ and equality constraint $\left(h_{s y s}(x)\right)$ represent the combination of all discipline level constraints. In this manner, the system level optimum $\left(f_{s y s}\left(x^{*}\right)\right)$ will satisfy all the constraints from all the disciplines. Repeating constraints are included only once in $g_{\text {sys }}(x)$ and $h_{\text {sys }}(x)$.

$$
\begin{gathered}
\min \left(f_{\text {sys }}(x)\right) \\
\text { subject to } g_{\text {sys }}(x) \leq 0, h_{\text {sys }}(x)=0 \\
l b_{\text {sys }} \leq x \leq u b_{\text {sys }}
\end{gathered}
$$

where as

$$
\begin{gathered}
f_{s y s}=e^{\left(\sum_{i=1}^{k} w_{i}\left(\frac{f_{i}(x)-f_{i}\left(x^{*}(i)\right)}{p r r_{i}}\right)^{2}\right)} \\
g_{\text {sys }}(x)=\left[\begin{array}{c}
g_{1}(x) \\
\vdots \\
g_{k}(x)
\end{array}\right] \text { and } h_{s y s}(x)=\left[\begin{array}{c}
h_{1}(x) \\
\vdots \\
h_{k}(x)
\end{array}\right]
\end{gathered}
$$

\section{Convergence of MLS Algorithm}

To demonstrate convergence on the system level optimization, the conditions (A1-A6), which guarantee convergence of each discipline level optimization, must also hold for the system level. These conditions are:

S1. $f_{\text {sys }}(x)$ is bounded below.

S2. $f_{\text {sys }}(x)$ is second-derivative continuous (i.e., the gradient and Hessian are continuous).

S3. $g_{s y s}(x)$ is second-derivative continuous (i.e., the gradient and Hessian are continuous).

S4. $h_{s y s}(x)$ is second-derivative continuous (i.e., the gradient and Hessian are continuous).

S5. $\left|g_{\text {sys }}(x)\right|<\infty \&\left|h_{\text {sys }}(x)\right|<\infty$ for $\left\{x: l b_{\text {sys }} \leq x \leq u b_{\text {sys }}\right\}$.

This section demonstrates that if A1-A6 are true, then the system level optimization satisfies property S1-S5.

\section{S1. $f_{\text {sys }}$ is bounded below.}

$$
f_{s y s}=e^{\left(\sum_{i=1}^{k} w_{i}\left(\frac{f_{i}(x)-f_{i}\left(x^{*}(i)\right)}{p r r_{i}}\right)^{2}\right)}
$$

$\left|f_{\text {sys }}\right| \geq e^{0}=1$. Thus, it is bounded below.

S2. $f_{\text {sys }}, \nabla f_{\text {sys }}$, and $\nabla^{2} f_{\text {sys }}$ are continuous.

(i) $\quad f_{\text {sys }}$ is continuous $\left(f_{\text {sys }}\right.$ maps $\left.\mathbb{R}^{n} \rightarrow \mathbb{R}\right)$

$f_{\text {sys }}=e^{\left(\sum_{i=1}^{k} w_{i}\left(\frac{f_{i}(x)-f_{i}\left(x^{*}(i)\right)}{p r r_{i}}\right)^{2}\right)}$

Define $c_{i}=\frac{w_{i}}{p r r_{i}^{2}}>0$ to simplify the notation

American Institute of Aeronautics and Astronautics 
$f_{\text {sys }}=e^{\left(\sum_{i=1}^{k} C_{i}\left(f_{i}(x)-f_{i}\left(x^{*}(i)\right)\right)^{2}\right)}$

Step 1.

$f_{i}\left(x^{*}(i)\right)$ is a constant, and $f_{i}(x)$ is a continuous function.

Thus, $f_{i}(x)-f_{i}\left(x^{*}(i)\right)$ is a continuous function.

Subsequently, $\left(f_{i}(x)-f_{i}\left(x^{*}(i)\right)\right)^{2}$ is also continuous.

Step 2.

$$
\boldsymbol{e}^{\sum_{i=1}^{k} C_{i}\left(f_{i}(x)-f_{i}\left(x^{*}(i)\right)\right)^{2}}=\sum_{m=0}^{\infty} \frac{\left(\sum_{i=1}^{k} C_{i}\left(f_{i}(x)-f_{i}\left(x^{*}(i)\right)\right)^{2}\right)^{m}}{m !}
$$

The summation of converging sequence of continuous function is also continuous.

Therefore, $f_{\text {sys }}$ is continuous.

(ii) $\quad \nabla f_{\text {sys }}$ is continuous $\left(\nabla f_{\text {sys }}\right.$ maps $\left.\mathbb{R}^{n} \rightarrow \mathbb{R}^{n}\right)$

$\nabla f_{\text {sys }}=\left\lceil f_{\text {sys }}\left(\sum_{i=1}^{k} 2 C_{i}\left(f_{i}(x)-f_{i}\left(x^{*}(i)\right)\right) \frac{\partial f_{i}(x)}{\partial x_{1}}\right) \ldots f_{\text {sys }}\left(\sum_{i=1}^{k} 2 C_{i}\left(f_{i}(x)-f_{i}\left(x^{*}(i)\right)\right) \frac{\partial f_{i}(x)}{\partial x_{n}}\right)\right\rceil$

$f_{\text {sys }}$ is continuous by $\mathrm{S} 2$ (i).

$f_{i}(x)$ and $\frac{\partial f_{i}(x)}{\partial x_{j}}$ are continuous by A2.

Subsequently, $\sum_{i=1}^{k} 2 C_{i}\left(f_{i}(x)-f_{i}\left(x^{*}(i)\right)\right) \frac{\partial f_{i}(x)}{\partial x_{j}}$ is continuous.

All columns of $\nabla f_{\text {sys }}$ are continuous.

Therefore, $\nabla f_{\text {sys }}$ is continuous.

(iii) $\quad \nabla^{2} f_{\text {sys }}$ is continuous

$\left(\nabla^{2} f_{\text {sys }}\right.$ maps $\left.\mathbb{R}^{n} \rightarrow \mathbb{R}^{n \times n}\right)$

$\nabla^{2} f_{s y s}=\left[\begin{array}{c}\frac{\partial \nabla f_{s y s}}{\partial x_{1}} \\ \vdots \\ \frac{\partial \nabla f_{s y s}}{\partial x_{n}}\end{array}\right]$

for each $\mathrm{j} \in[1 \ldots \mathrm{n}]$

$$
\begin{aligned}
\frac{\partial \nabla f_{\text {sys }}}{\partial x_{j}}=\left[\frac{\partial f_{\text {sys }}}{\partial x_{j}}\right. & \left(\sum_{i=1}^{k} 2 C_{i}\left(f_{i}(x)-f_{i}\left(x^{*}(i)\right)\right) \frac{\partial f_{i}(x)}{\partial x_{1}}\right)+f_{s y s}\left(\sum_{i=1}^{k} 2 C_{i} \frac{\partial f_{i}(x)}{\partial x_{j}} \cdot \frac{\partial f_{i}(x)}{\partial x_{1}}\right) \\
& +f_{s y s}\left(\sum_{i=1}^{k} 2 C_{i}\left(f_{i}(x)-f_{i}\left(x^{*}(i)\right)\right) \frac{\partial^{2} f_{i}(x)}{\partial x_{1} \partial x_{j}}\right) \cdots \frac{\partial f_{s y s}}{\partial x_{j}}\left(\sum_{i=1}^{k} 2 C_{i}\left(f_{i}(x)-f_{i}\left(x^{*}(i)\right)\right) \frac{\partial f_{i}(x)}{\partial x_{j}}\right) \\
& +f_{s y s}\left(\sum_{i=1}^{k} 2 C_{i}\left(\frac{\partial f_{i}(x)}{\partial x_{j}}\right)^{2}\right) \\
& +f_{\text {sys }}\left(\sum_{i=1}^{k} 2 C_{i}\left(f_{i}(x)-f_{i}\left(x^{*}(i)\right)\right) \frac{\partial^{2} f_{i}(x)}{\partial x_{j}{ }^{2}}\right) \cdots \frac{\partial f_{s y s}}{\partial x_{j}}\left(\sum_{i=1}^{k} 2 C_{i}\left(f_{i}(x)-f_{i}\left(x^{*}(i)\right)\right) \frac{\partial f_{i}(x)}{\partial x_{n}}\right) \\
& \left.+f_{s y s}\left(\sum_{i=1}^{k} 2 C_{i} \frac{\partial f_{i}(x)}{\partial x_{j}} \cdot \frac{\partial f_{i}(x)}{\partial x_{n}}\right)+f_{s y s}\left(\sum_{i=1}^{k} 2 C_{i}\left(f_{i}(x)-f_{i}\left(x^{*}(i)\right)\right) \frac{\partial^{2} f_{i}(x)}{\partial x_{j} \partial x_{n}}\right)\right]
\end{aligned}
$$


$f_{s y s}, \frac{\partial f_{s y s}}{\partial x_{j}}$ is continuous by S2(i) and S2(ii).

$f_{i}(x), \frac{\partial f_{i}(x)}{\partial x_{j}}, \frac{\partial^{2} f_{i}(x)}{\partial x_{i} \partial x_{j}}$ are continuous by A2.

All rows of $\nabla^{2} f_{s y s}$ are continuous.

Therefore $\nabla^{2} f_{\text {sys }}$ is continuous.

S3 \& S4. $\boldsymbol{g}_{\text {sys }}(\boldsymbol{x})$ is second derivative continuous, and $\boldsymbol{h}_{\text {sys }}(\boldsymbol{x})$ is second derivative continuous.

$g_{\text {sys }}(x)$ and $h_{\text {sys }}(x)$ are the collection of second derivative continuous discipline level inequality and equality constraints $g_{i}(x)$ and $h_{i}(x)$. By A3 and A4, $g_{i}(x)$ and $h_{i}(x)$ are second derivative continuous.

Therefore, $g_{s y s}(x)$ and $h_{s y s}(x)$ are second derivative continuous.

S5. $\left|g_{\text {sys }}(x)\right|<\infty$ and $\left|h_{s y s}(x)\right|<\infty$ for $\left\{x: l b_{\text {sys }} \leq x \leq u b_{\text {sys }}\right\}$

$g_{\text {sys }}(x)$ and $h_{\text {sys }}(x)$ are the collection of second derivative continuous discipline level inequality and equality constraints $g_{i}(x)$ and $h_{i}(x) . g_{i}(x)$ and $h_{i}(x)$ are bounded for $\left\{x: l b_{i} \leq x \leq u b_{i}\right\}$.

By definition $\left(l b_{i} \leq l b_{\text {sys }} \leq u b_{\text {sys }} \leq u b_{i}\right)$

Therefore, $\left|g_{\text {sys }}(x)\right|<\infty$ and $\left|h_{\text {sys }}(x)\right|<\infty$ are bounded for $\left\{x: l b_{s y s} \leq x \leq u b_{\text {sys }}\right\}$.

\section{Analytical Example}

To demonstrate the performance of the MLS algorithm, the authors solved a multi-discipline optimization example that was based on the Sellar Problem ${ }^{43}$. The original optimization statement of the Sellar problem is

$$
\begin{gathered}
\min \left(f_{\text {obj }}\left(z_{1}, z_{2}, x_{1}, y_{1}, y_{2}\right)=x_{1}^{2}+z_{2}+y_{1}+e^{-y_{2}}\right) \\
\text { subject to } \\
g_{1}(x)=\frac{y_{2}}{24}-1 \leq 0 \\
g_{2}(x)=1-\frac{y_{1}}{3.16} \leq 0 \\
y_{1}\left(z_{1}, z_{2}, x_{1}, y_{2}\right)=z_{1}^{2}+x_{1}+z_{2}-0.2 y_{2} \\
y_{2}\left(z_{1}, z_{2}, y_{1}\right)=\sqrt{y_{1}}+z_{1}+z_{2} \\
{\left[\begin{array}{lll}
-10 & 0
\end{array}\right] \leq\left[\begin{array}{lll}
z_{1}, z_{2}, x_{1}
\end{array}\right] \leq\left[\begin{array}{lll}
10 & 10 & 10
\end{array}\right]}
\end{gathered}
$$

In the past, the Sellar problem has been used in Refs. 43 for demonstrating the performance of a Collaborative Optimization (CO) algorithm. Due to the interdependency between $f_{o b j}, y_{1}$, and $y_{2}$, a sub-optimization is solved with the CO. MLS algorithm defines three disciplines with the functions of the Sellar problem. Each discipline optimization is solved as a separate CO.

The original Sellar problem is used as the first discipline in the MLS example. The construction remains the same in the other two disciplines optimization statements, but functions $y_{1}$ and $y_{2}$ comprise each objective functions respectively. Within each discipline optimization, $y_{1}$ and $y_{2}$ are evaluated by solving a sub-optimization problem which minimizes the Error between estimate of $y_{1}$ and $y_{2}$ and the corresponding final values.

$$
\text { Error }=\left(y_{1}^{*}-y_{1}\right)^{2}+\left(y_{2}^{*}-y_{2}\right)^{2} \quad y_{1}^{*}=z_{1}^{2}+x_{1}+z_{2}-0.2 y_{2} \quad y_{2}^{*}=\sqrt{y_{1}}+z_{1}+z_{2}
$$

The MLS algorithm uses information from the discipline level optimizations to update the system level optimization. This algorithm can also be used for evaluating the Pareto front by assigning various weights to the system level objective function.

Table 1 summarizes MLS optimization statement based on the Sellar problem. It lists all disciplines objective functions, a system level objective function, inequality constraints, and upper and lower bounds of design variables. $p r_{y 1}$ is several orders of magnitude smaller than the other prrs. Therefore, only small change can be identified in $y_{1}$. 
Upper bound $(u b)$, lower bound $(l b)$, inequality constraints $(g(x))$, and equality constraints $(h(x))$ are identical for all discipline-level and a system-level optimization for this problem.

Table 1. Definitions of MLS Optimization based on the Sellar problem.

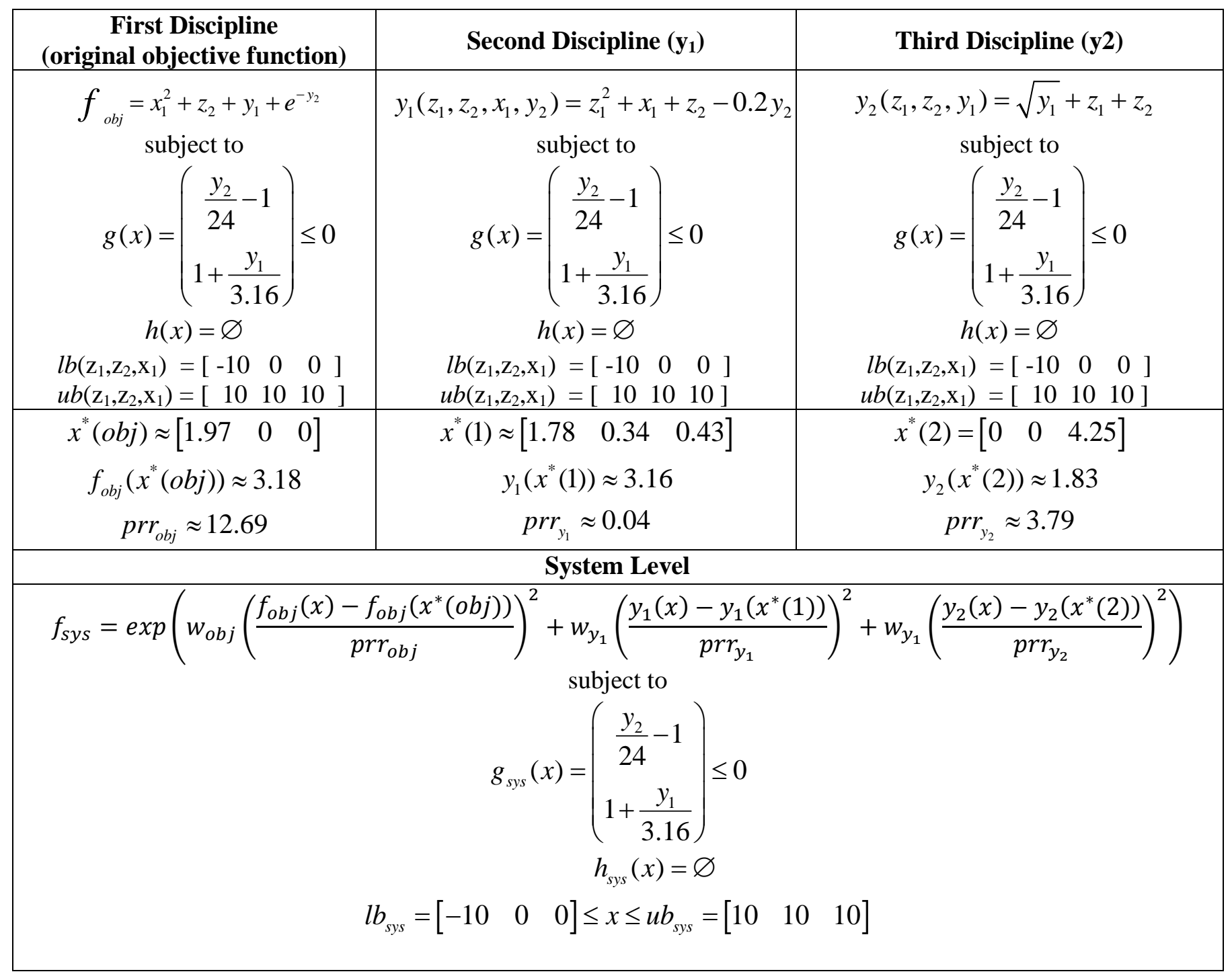

To assess the performance of the MLS algorithm, a Monte Carlo (MC) simulation was conducted. 248625 numbers of Monte Carlo simulations were considered, and the simulation resulted 176883 feasible points and a Pareto front with 113 non-dominated points. In Table 2, The MLS algorithm result is compared with published data using a Collaborative Optimization (CO) of the original objective function. ${ }^{44}$

Table 2. Results Comparison between Published Data

\begin{tabular}{|c|c|c|c|c|c|c|c|}
\hline & $z_{1}$ & $z_{2}$ & $x_{1}$ & $f_{o b j}$ & $y_{1}$ & $y_{2}$ & Note \\
\hline Published Data using CO & 1.978 & 0.000 & 0.000 & 3.183 & 3.160 & 3.756 & - \\
\hline MLS & 1.551 & 0.000 & 1.419 & 5.209 & 3.160 & 3.329 & Equal weights for $f_{o b j}, y 1$, and $y 2$ \\
\hline $\begin{array}{c}\text { Change in Value } \\
\text { (\% of } p r r \text { ) }\end{array}$ & - & - & - & $15.96 \%$ & $-1.16 \%$ & $-11.25 \%$ & - \\
\hline
\end{tabular}


The new MLS algorithm performs as authors has expected. The value of $f_{o b j}$ increases with the MLS algorithm because the algorithm no longer solely minimizes $f_{o b j}$. The value of $y_{2}$ decreases by $11 \%$ of $p r r_{y 1}$. The value of $y_{1}$ decreases by less than $10^{-4}$. As mentioned above, small reduction in the $y_{2}$ can be attributed to small $p r r_{y 2}$.

Fig. 3 shows the 3-dimensional numerical Pareto front of the Sellar problem and the converged points in relation to the Pareto front. The converged points are in neighbor of the Pareto front identified by the MC simulation. This shows that the algorithm converges on the Pareto front.

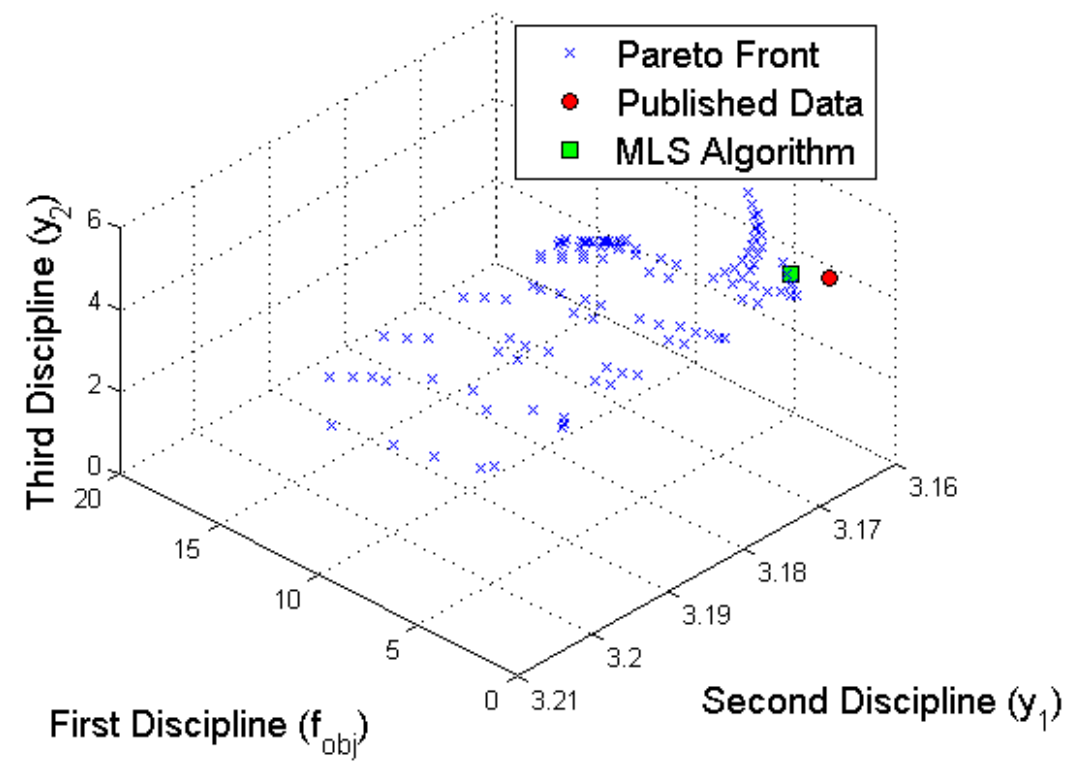

Figure 3. Numerical Pareto front of the Sellar Problem.

Fig. 4 is a 2-dimensional projection of the numerical Pareto front of $f_{o b j}$ and $y_{2}$. As mentioned above the range of $y_{1}$ axis is orders of magnitude smaller than that of $f_{o b j}$.

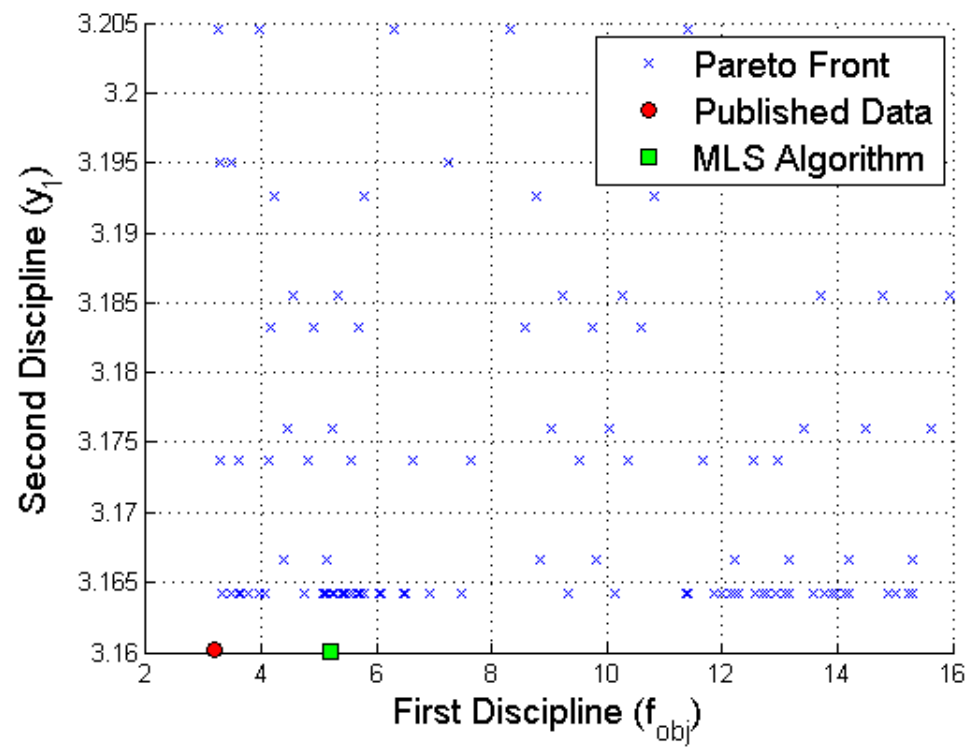

Figure 4. Numerical Pareto front of the Sellar Problem $y_{1}$ vs. $f_{o b j}$.

American Institute of Aeronautics and Astronautics 
Fig. 5 highlights the main capabilities of the MLS algorithm. The 2-dimensional projection of the numerical Pareto front of $f_{o b j}$ and $y_{2}$ shows the non-convexity of the Pareto front and tradeoff between $f_{o b j}$ and $y_{2}$. The MLS algorithm is capable of controlling convergence on the Pareto front while CO can only converge onto $f_{\text {obj }}$ minimum.

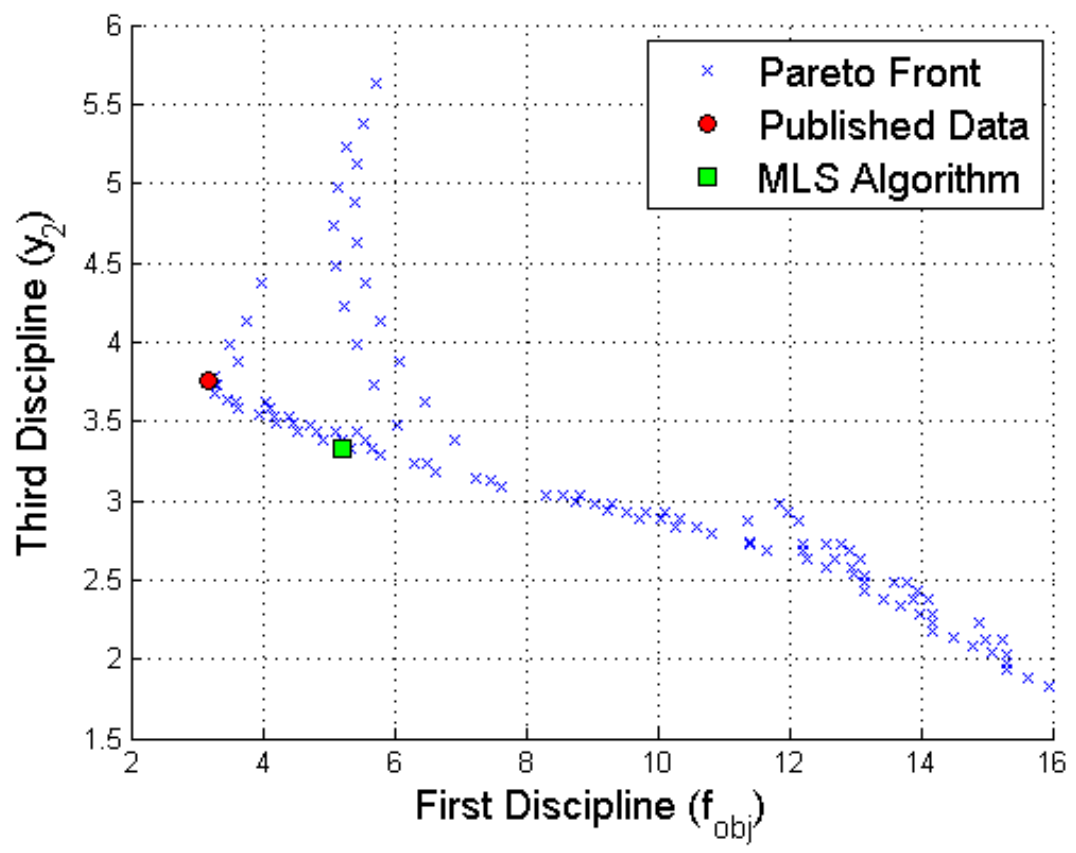

Figure 5. Numerical Pareto front of the Sellar Problem $f_{\text {obj }}$ vs. $y_{2}$.

Fig. 6 is a 2-dimensional projection of the numerical Pareto front of $y_{2}$ and $y_{1}$.

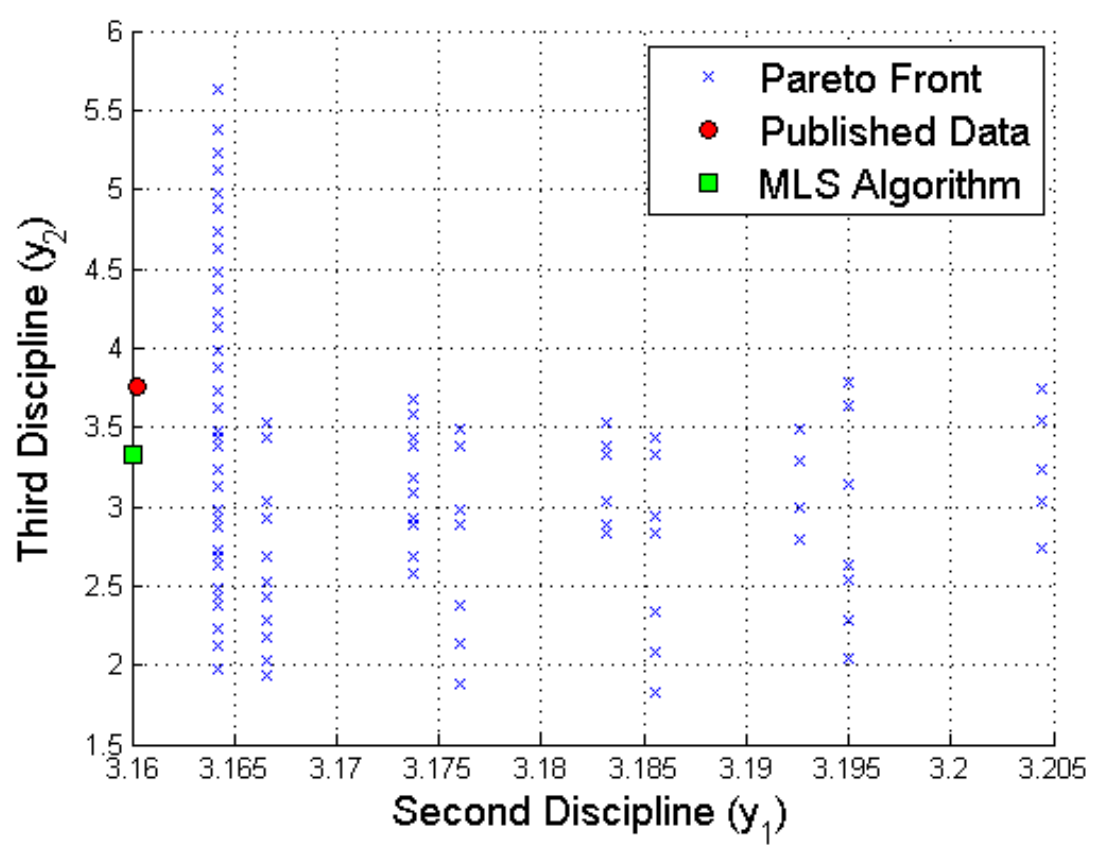

Figure 6. Numerical Pareto front of the Sellar Problem $y_{2}$ vs. $y_{1}$.

American Institute of Aeronautics and Astronautics 


\section{Ship Analysis.}

To demonstrate how the new algorithm can be used for analyzing a complex engineering system, a design analysis is performed for a general cargo ship. This analysis contains multiple objectives, but the interdependency and the tradeoff of these objectives cannot be easily identified because of complex relationship between design variables and the objectives. The objectives are associated with the lightship weight $(L W)$, the transportation cost (TC), and the annual cargo $(A C) .{ }^{42}$ The optimization statement is written to minimize $L W$ and $T C$ and to maximize $A C$, which is equivalent to minimization of negative $A C$. Table 3 defines objective functions, design variables, inequality constraints, and various parameters that are required to compute objective functions. The problem has six design variables: Length $(L)$, Beam $(B)$, Depth $(D)$, Draft $(T)$, Block Coefficient $\left(C_{B}\right)$, and Ship Speed $\left(\mathrm{V}_{\mathrm{k}}\right)$, and eight inequality constraints. The system level objectives function is expressed as:

$$
f_{s y s}=\exp \left(w_{L W}\left(\frac{L W(x)-L W\left(x^{*}(l w)\right)}{p r r_{L W}}\right)^{2}+w_{T C}\left(\frac{T C(x)-T C\left(x^{*}(t c)\right)}{p r r_{T C}}\right)^{2}+w_{A C}\left(\frac{-A C(x)+A C\left(x^{*}(a c)\right)}{p r r_{A C}}\right)^{2}\right)
$$

where $\mathrm{w}_{\mathrm{Lw}}, \mathrm{w}_{\mathrm{TC}}$, and $\mathrm{w}_{\mathrm{AC}}$ are the weights assigned to each one of the three disciplines. Technical details about the mathematical models used in this ship analysis are present in Ref. 42. and summarized in Table 3.

Table 3. Technical background of ship example ${ }^{42}$.

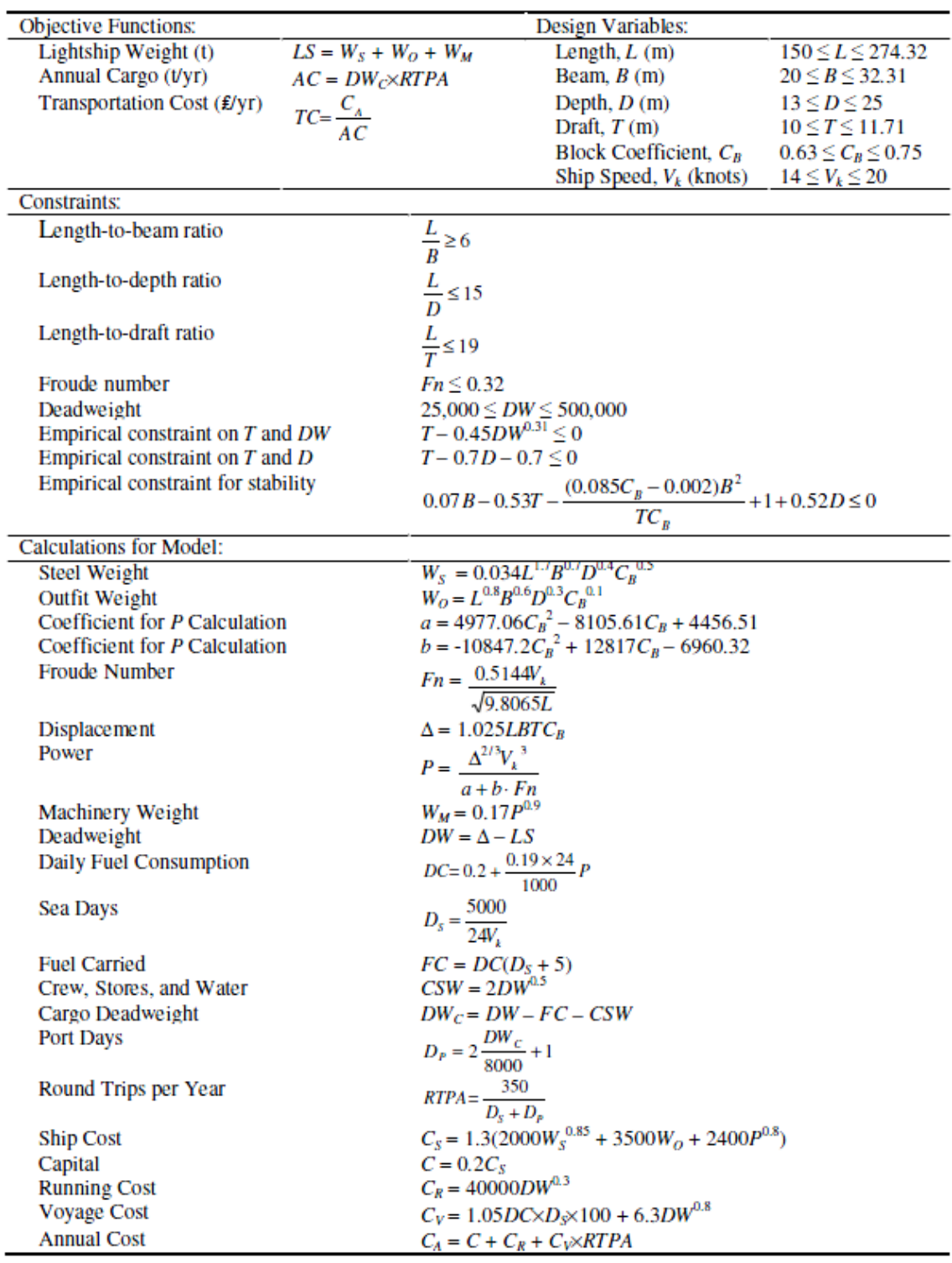

American Institute of Aeronautics and Astronautics 
The numerical Pareto front is constructed by the new MLS algorithm with the exhaustive permutation of [0 0.1 $\left.\begin{array}{lllllllllll}0.2 & 0.25 & 0.3 & 0.4 & 0.5 & 0.6 & 0.7 & 0.75 & 0.8 & 0.9 & 1\end{array}\right]$ on the system level objective function weights: $\mathrm{w}_{\mathrm{Lw}}, \mathrm{w}_{\mathrm{TC}}$, and $\mathrm{W}_{\mathrm{AC}}$. This permutation produces $2197\left(=11^{3}\right)$ system level objective functions for each initial point. The authors tested the algorithm with three different initial points resulting in 6591 $\left(=11^{3} \times 3\right)$ MLS optimization statements.

Out of 6591 converged points, 4434 points are non-dominated. 2157 dominated points are expected because of the redundant permutation of weights and the optimization statements with different initial points converging on the same point on the Pareto front. The result shows that the MLS algorithm is capable and useful in identifying the Pareto front for general engineering design problems.

For plotting purposes, all objective functions are non-dimensionalized, and the non-dimensionalized factors are 10300 (t) for LW, -548000 (t/yr) for AC, and 9.93 ( $£ / y r)$ for TC. The non-dimensionalized prrs are 12.068 for $L W$, 9.734 for $A C$, and 3.294 for TC. Table 4 summarizes the results when equal weights are assigned to all three disciplines. The corresponding optimum is presented in the Pareto front plots as a point of reference to demonstrate the compromise between the mutually competing discipline objectives.

Table 4. Converged Point with Equal Weights.

\begin{tabular}{|c|c|c|c|c|c|c|c|c|}
\hline $\mathrm{L}(\mathrm{m})$ & $\mathrm{B}(\mathrm{m})$ & $\mathrm{D}(\mathrm{m})$ & $\mathrm{T}(\mathrm{m})$ & $\mathrm{C}_{\mathrm{B}}$ & $\mathrm{V}_{\mathrm{k}}$ (knots) & $\mathrm{TC}$ & $-\mathrm{AC}$ & $\mathrm{LW}$ \\
\hline 215.39 & 32.31 & 15.73 & 11.71 & 0.75 & 14.00 & 8.53 & -11.31 & 10.81 \\
\hline
\end{tabular}

Figs. 7-10 show the Pareto front constructed using the MLS algorithm. Fig. 7 shows a 3-dimensional scatter plot of non-dominated points found by the MLS algorithms. Figs. 8-10 comprise two dimensional projections of the Pareto front.

Table 4 summarizes the six design variables and the objective functions when equal weights are considered in the definition of the system level objective function. It can be observed that assigning equal weight to each discipline leads to a balanced design and avoids the region of extreme values for the objective functions.

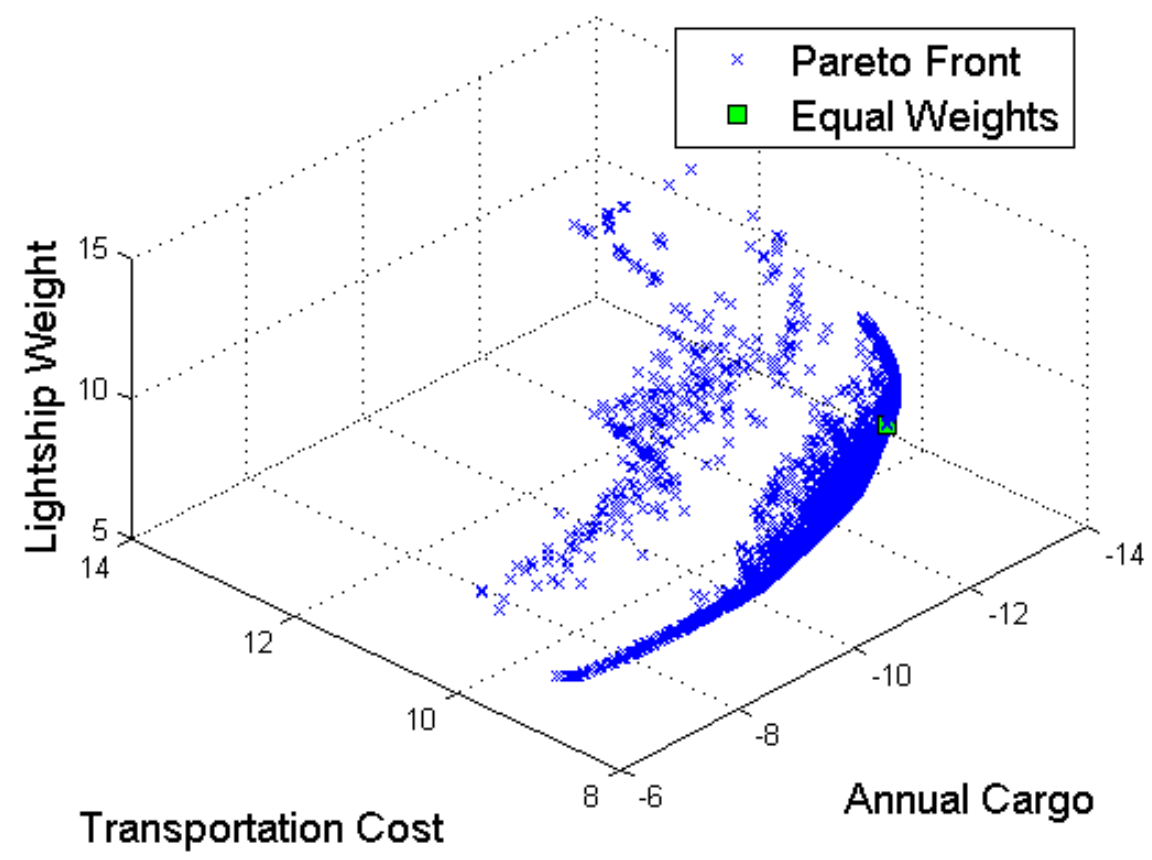

Figure 7. Container Ship Problem Pareto Front. 


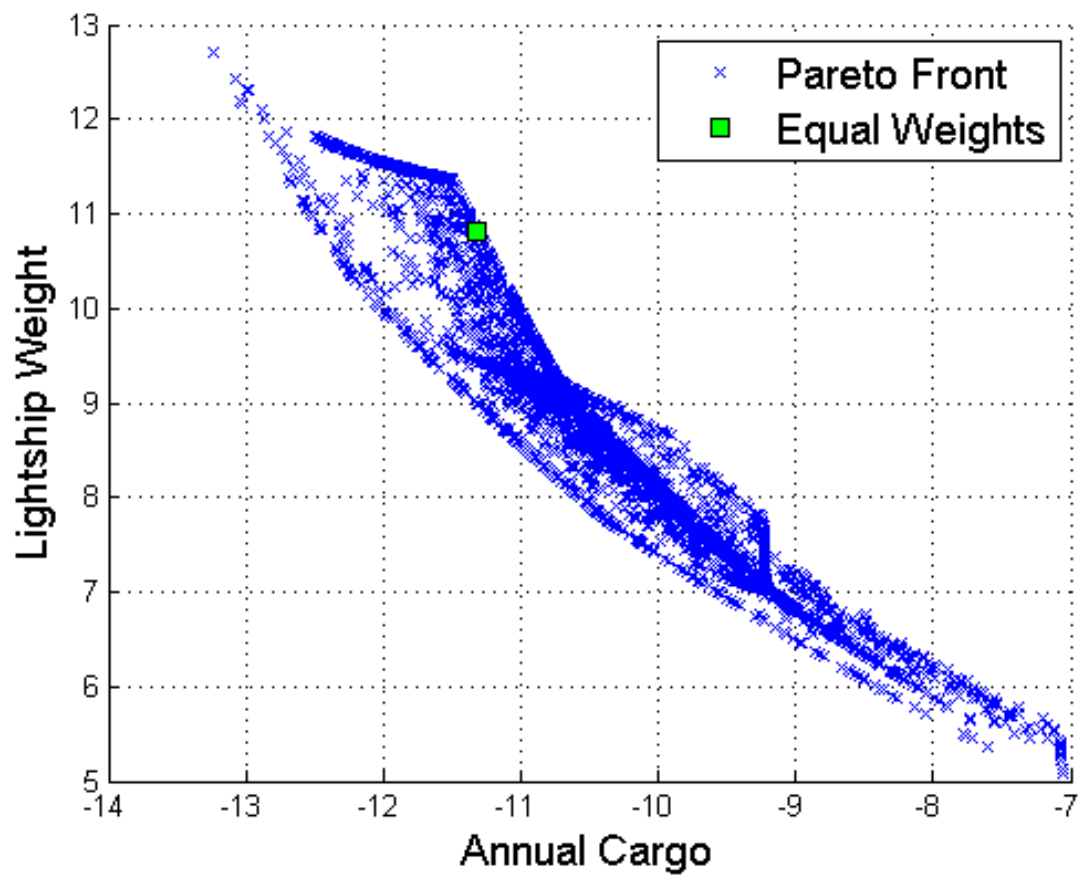

Figure 8. Annual Cargo vs. Lightship Weight.

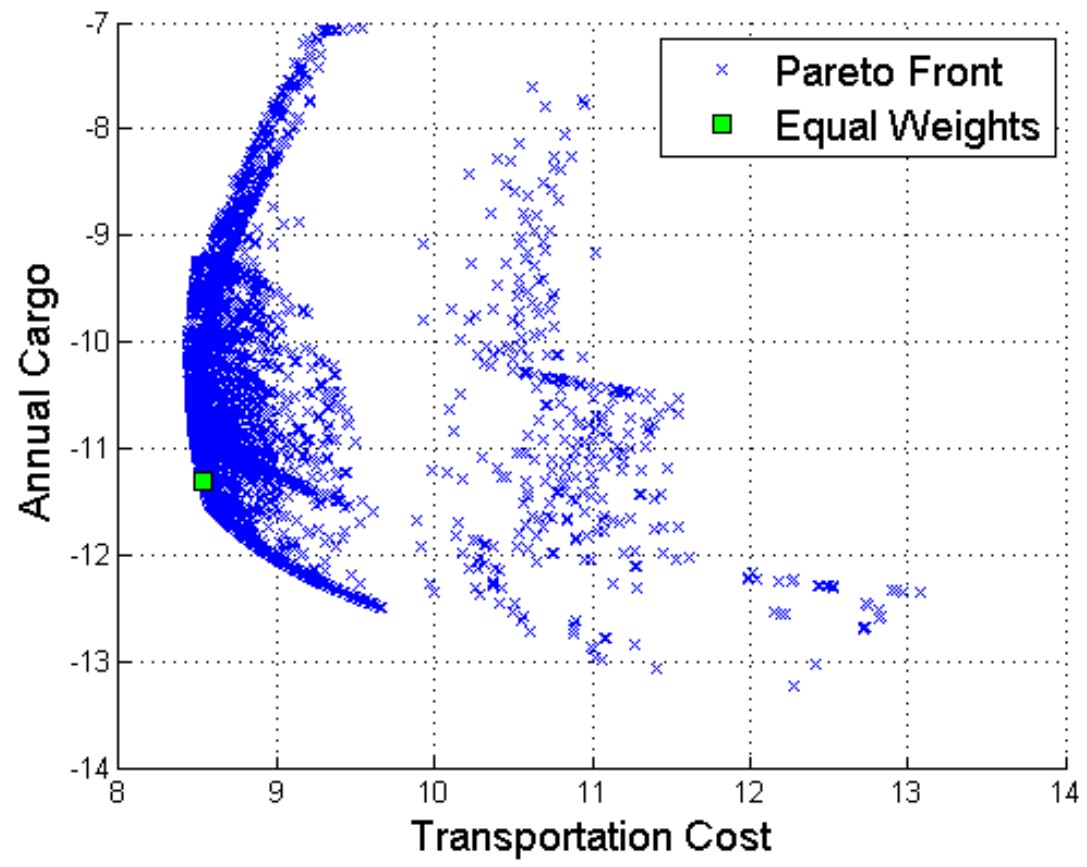

Figure 9. Transportation Cost vs. -Annual Cargo.

American Institute of Aeronautics and Astronautics 


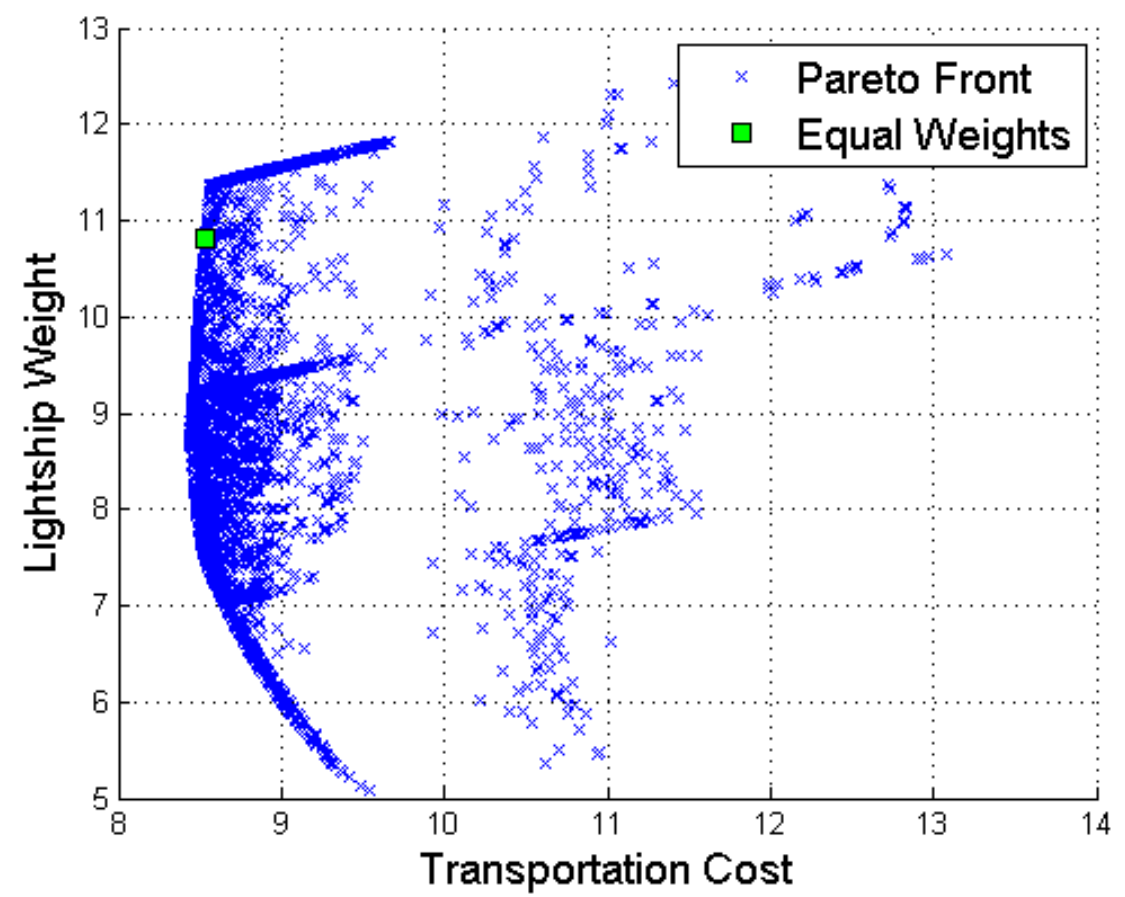

Figure 10. Transportation Cost vs. Lightship Weight.

Unlike single objective optimizations, which identify one optimal point, the MLS algorithm can be used for identifying the non-dominated set. The capability to construct the Pareto front without conducting a MC analysis is immensely useful during decision making processes when the interdependency and the tradeoff between multiple objectives are not intuitive. The successful construction of the Pareto front of the ship analysis confirms the utility of the MLS algorithm in general engineering design problems.

\section{Summary}

An MLS optimization algorithm and its mathematical proof of convergence are presented. The structure of the algorithm is comprised by a system level optimization and multiple discipline level optimizations for simultaneously minimizing all discipline level objective functions. The algorithm uses the system level optimization for coordinating the interaction and the exchange of information among multiple discipline level optimizations. Mathematical convergence of the system level is proven given that the discipline level optimization problems are well conditioned. The Sellar problem, which has been used in the past for evaluating CO algorithms, provides the basis for defining a multi-level optimization problem with three discipline level CO statements. The new MLS algorithm is used for conducting the analysis, and the results demonstrate the capabilities of the new algorithm for balancing competing objectives, facilitating the exchange of information between disciplines, and being able to identify a non-convex Pareto front. The ship analysis demonstrates that the algorithm can be useful for solving general engineering design problems. 


\section{References}

1. Fletcher. R. Practical Methods of Optimization. Wiley-Interscience, New York, NY, 1987.

2. Statnikov, R.B. and Matusov, J.B., Multicriteria Optimization and Engineering. Chapman and Hall, 1995.

3. Sen, P. and Yang, J.B., Multiple Criteria Decision Support in Engineering Design. Springer, New York, 1998.

4. $\quad$ Papalambros, P. Y. and Wilde, D. J., Principles of Optimal Design. 2000.

5. Floudas, C. A. and Pardalos, P. M., Encyclopedia of Optimization. Kluwer Academic, 2001.

6. Kendall, P. M. H. “A Theory of Optimum Ship Size,” Journal of Transport Economics and Policy, 6:128-146, 1972.

7. Jansson, J. O. and Shneerson, D., “The Optimal Ship Size,” Journal of Transport Economics and Policy, 16:217-238, 1982.

8. Cox, S. E., Haftka, R. T., et al. "A Comparison of Global Optimization Methods for the Design of a High-speed Civil Transport,” Journal of Global Optimization, 21(4):415-432, 2001.

9. Moraes, H. B., Vasconcellos,J., and Almeida, M. P.M., "Multiple Criteria Optimization Applied to High Speed Catamaran Preliminary Design,” Ocean Engineering, 34 (1):133-147, 2007.

10. Kim, H. M., Kokkolaras, M. et al. “Target Cascading in Vehicle Redesign: A class VI Truck Study,” International Journal of Vehicle Design, 29(3): 199-225, 2002.

11. Sinha, K. "Reliability-based Multiobjective Optimization for Automotive Crashworthiness and Occupant Safety," Structural and Multidisciplinary Optimization, 33(3):255-268, 2007.

12. Venkayya, V. B. “Optimality criteria: A Basis for Multidisciplinary Design Optimization,” Computational Mechanics, 5(1):1-21, 1989.

13. Kim, I. Y. and de Weck, O. L., “Adaptive Weighted-sum Method for Bi-Objective Optimization: Pareto Front Generation,” Structural and Multi-disciplinary Optimization, 29(2):149-158, 2005.

14. Parashar, S. and Bloebaum, C. L., "Multi-Objective Genetic Algorithm Concurrent Subspace Optimization (MOGACSSO) for Multidisciplinary Design,” In 47th AIAA/ASME/ASCE/AHS/ASC Structures, Structural Dynamics, and Materials Conference, pages 1-11, 2006.

15. Moles,C. G., Mendes, P., and Banga, J. R., "Parameter Estimation in Biochemical Pathways: A Comparison of Global Optimization Methods,” Genome Research, 13 (11):2467-2474, 2003.

16. Lewis, K. “Multidisciplinary Design Optimization,” Aerospace America, 40(12): 42, 2002.

17. Idahosa, U., Golubev, V. V., and Balabanov, V. O., "Application of Distributed Automated MDO Environment to Aero/Acoustic Shape Optimization of a Fan Blade,” In 11th AIAA/CEAS Aeroacoustics Conference (26th Aeroacoustics Conference), 2005.

18. Berends, J., van Tooren, M. J. L., and Belo, D. N. V., “A Distributed Multi-Disciplinary Optimisation of a Blended Wing Body UAV using a Multi-agent Task Environment,” In 47th AIAA/ASME/ASCE/AHS/ASC Structures, Structural Dynamics, and Materials Conference, 2006.

19. Jouhaud, J. C., Sagaut, P., Montagnac, M., and Laurenceau, J., “A Surrogate-model Based Multidisciplinary Shape Optimization Method with Application to a 2D Subsonic Airfoil,” Computers and Fluids, 36(3):520-529, 2007.

20. Frank, P.D., Booker, A.J., Caudell, T.P., and Healy, M.J., "A Comparison of Optimization and Search methods for Multidisciplinary Design,” AIAA Paper 92-4827, Sept. 1992.

21. Alexandrov, N. M., and Hussaini, M. Y., "Multidisciplinary Design Optimization: State of the Art," Society for Industrial \& Applied, 1997.

22. Vianese, J., Cary, N. C., “Multidisciplinary Optimization of Naval Ship Design and Mission Effectiveness,” Defense Technical Information Center, 2004.

23. Morris, A. and Arendsen, P., “Mob-a European Project on Multidisciplinary Design Optimisation,” 2004.

24. Sun, J., Zhang, G., Vlahopoulos, N., and Hong, S,. "Multi-Disciplinary Design Optimization Under Uncertainty for Thermal Protection System Applications,” In AIAA, number 2006-7002-547, 2006.

25. Vlahopoulos, N. and He, Z. J., “Application of a Multi-Discipline Design Optimization Approach for Designing the Thermal Protection System of an Apollo-type Vehicle Under Uncertainty,” Proceedings of 2008 JANNAF Meeting, Orlando, Florida, 2008.

26. Yi, S. I., Shin, J. K., and Park, G. J., “Comparison of MDO Methods with Mathematical Examples,” Structural and Multidisciplinary Optimization, 35(5):391-402, 2008.

27. Cramer,E. J., Dennis, J. E., Frank, P. D., Lewis, R. M., and Shubin, G. R., "Problem Formulation for Multidisciplinary Optimization,” SIAM Journal on Optimization, 4(4):754-776, 1994.

28. Giesing, J. P. and Jean-Francois, M. B., “A Summary of Industry MDO applications and Needs,” In Symposium on Multidisciplinary Analysis and Optimization, 1998.

29. Hulme, K. F. and Bloebaum, C. L., "A Simulation-based Comparison of Multidisciplinary Design Optimization Solution Strategies Using CASCADE,” Structural and Multidisciplinary Optimization, 19(1):17-35, 2000.

30. Kim, H. M., Michelena, N. F., Papalambros, P.Y., and Jiang, Tao., “Target Cascading in Optimal System Design.” Journal of Mechanical Design vol. 125, no. 3, pp. 474-480, 2003.

31. Michelena, N., Louca, L., et al. "Design of an Advanced Heavy Tactical Truck: A Target Cascading Case Study,” SAE TRANSACTIONS, 110(2):457-468, 2001 
32. Garcia-Palomares,U. M., Burguillo-Rial, J. C., and Gonzalez-Castano, F. J., "Explicit Gradient Information in Multiobjective Optimization," Operations Research Letters, vol. 36, pp. 722-725, 2008

33. Balling, R. and Sobieszczanski-Sobieski, J., "An Algorithm for Solving the System-Level Problem in Multilevel Optimization," Institue for Computer Application in Science and Engineering NASA Langley Research Center, Hampton, 1994.

34. Marlor, R. and Arora, J., "Survey of Multi-Objective Optimization Methods for Engineering," Struct Multidisc Optim, vol. 26, pp. 369-395, 2004.

35. Hwang, C.L. and Yoon, K., "Multiple Attribute Decision Making: Methods and Applications," Springer-Verlag, New York, 1981.

36. Lai, Y., Liu, T. and Hwang, C., "TOPSIS for MODM," European Journal of Operational Research, vol. 76, no. 3, pp. 486500, 1994.

37. Akrotirianakis, I. and Rustem, B., "A Globally Convergent Interior Point Algorithm for Non-linear Programming Problems," Department of Computing Imperial College, London.

38. Griva, I., Shanno, D. F. and Vanderbei, R. J., "Convergence Analysis of a Primal-Dual Interior-Point Method for Nonlinear Programming," 2004.

39. Wright. S., "Convergence of SQP-like Methods for Constrained Optimization," SIAM J. Control and Optimization, vol. 27, no. 1 , pp. 13-26, 1989.

40. Powell, M., "On the Global Convergence of Trust Region Algorithms for Unconstrained Minimization," Mathematicl Programming, vol. 29, pp. 297-303, 1984.

41. El-Alem, M., "A Global Convergence Theory for the Celis-Dennis-Tapia Trust-region Algorithms for Constrained Optimization," SIAM J. Numer. Anal., vol. 28, no. 1, pp. 266-290, 1991.

42. Hannapel, S. E. and Vlahopoulos, N., "Robust and Reliable Multidiscipline Ship Design," in 13th AIAA/ISSMO Multidisciplinary Analysis Optimization Conference, Fort Worth, 2010.

43. Sellar, R. S., Batill, S. M., and Renaud, J. E., "Response Surface Based, Concurrent Subspace Optimization for Multidisciplinary System Design,” Proceedings References 79 of the 34th AIAA Aerospace Sciences Meeting and Exhibit, Reno, NV, January 1996.

44. "OpenMDAO," [Online]. Available: http://openmdao.org/dev_docs/mdao/intro.html. [Accessed 25 January 2012]. 